\title{
Cutaneous sarcoidosis and secondary open-angle glaucoma in a patient: case report and literature review*
}

\author{
Qiancheng Deng ${ }^{1}$ \\ Shengbo Yang ${ }^{1}$
}

\author{
Shu Ding ${ }^{1}$ \\ JinHua Huang ${ }^{1}$
}

DOI: http:/ /dx.doi.org/10.1590/abd1806-4841.20175368

\begin{abstract}
The current report presents the case of a 41-year-old male patient with a two-month history of asthenopia and plaques in the frontotemporal region. Computed tomography revealed bilateral hilar and mediastinal lymphadenopathy. Ophthalmological examination showed elevated intraocular pressure. Skin biopsy demonstrated aa dermal inflammatory infiltrate composed mainly of epithelioid cells and a few multinucleated giant cells, but no obvious lymphocytes. Findings of thorough physical examinations and auxiliary examinations suggested the presence of cutaneous sarcoidosis and secondary open-angle glaucoma. Treatment consisted mainly of oral methylprednisolone. Skin lesions, bilateral hilar, and mediastinal lymphadenopathy resolved completely. Cutaneous sarcoidosis is often accompanied by extracutaneous organ involvement. Dermatologists must be aware of the disease's extracutaneous manifestations to ensure accurate diagnosis for further treatments.
\end{abstract}

Keywords: Clinical diagnosis; Glaucoma; Sarcoidosis

\section{INTRODUCTION}

Sarcoidosis is a multisystem granulomatous inflammatory disease whose exact pathogenesis is unknown. It may be associated with alteration in the cellular immune response after exposure to an environmental or a persistent infectious hazard. ${ }^{1,2}$ The lungs and lymphatic system are most commonly involved and it usually manifests mediastinal or hilar lymphadenopathy. ${ }^{1,3}$ Skin is the second most commonly involved organ after the lung and skin involvement occurs in $25 \%$ of cases. ${ }^{1,4}$ Ocular manifestations are present in $30 \%$ of cases. ${ }^{5}$ Here , we present an uncommon case of cutaneous sarcoidosis with secondary open-angle glaucoma.

\section{CASE REPORT}

A 41-year-old male patient presented to our dermatology department with the complaint of a two-month history of asthenopia and plaques in the frontotemporal region (Figure 1). He reported no pruritus and denied fever, cough, expectoration, hemoptysis, night sweats, anhelation, chest pain, and other symptoms.

Physical examination revealed normal vital signs. There were multiple, non-tender, infiltrated red plaques of varied sizes and irregular shapes in the frontotemporal region. Ophthalmological examination manifested bilateral eye conjunctival congestion (Figure 2). Intraocular pressure (IOP) measured $49 \mathrm{mmHg}$ OD and
$37 \mathrm{mmHg}$ OS. Fundus examination revealed cup-to-disc ratio of 0.4 right and 0.3 left. Other systemic examinations revealed no abnormalities.

Laboratory investigations showed normal blood count and liver function. Renal function manifested a uric acid level of 514 $\mu \mathrm{mol} / \mathrm{l}$. C-reactive protein was slightly elevated. Erythrocyte sedimentation rate revealed normal. Anti-nuclear antibody tested positive. Other tests, including ANCA, anti-DS DNA, anti-SM, anti-SSA, and anti-SSB, were negative. Computed tomography (CT) revealed bilateral hilar and mediastinal lymphadenopathy. Pulmonary function test showed normal. Positive tuberculosis antibody-IgG and negative tuberculin test were seen.

Histopathology of skin biopsy revealed a dermal inflammatory infiltrate composed mainly of epithelioid cells and a few multinucleated giant cells, but no obvious lymphocytes (Figure 3). Special stain for acid-fast bacterial and fungal organisms was negative. Mediastinal lymphadenopathy biopsy revealed piles of small lymphocyte grouping and a few epithelioid macrophages,, no malignant cells and apparent giant cells, which tended to granulomatous inflammation (Figure 4).

We diagnosed cutaneous sarcoidosis with secondary open-angle glaucoma. Initial treatment consisted of oral meth-

Received on 12.11.2015

Approved by the Advisory Board and accepted for publication on 02.02.2016

* Work performed at the Department of Dermatology, Third Xiangya Hospital, Central South University - Changsha, China. Financial support: none.

Conflict of interest: none.

1 Department of Dermatology, Third Xiangya Hospital, Central South University, Changsha, China.

(O2017 by Anais Brasileiros de Dermatologia 
ylprednisolone $(24 \mathrm{mg} /$ daily), topical halometasone cream, and brinzolamide and brimonidine eye drops. Within five days or so, the skin lesions gradually softened and flattened, showing a dramatic response. Asthenopia and conjunctival congestion symptoms disap-

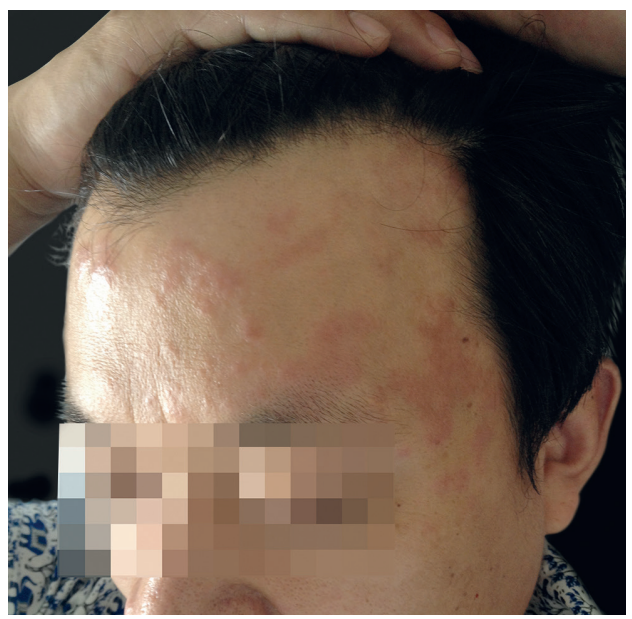

Figure 1: Numerous plaques of varied sizes in the frontotemporal region

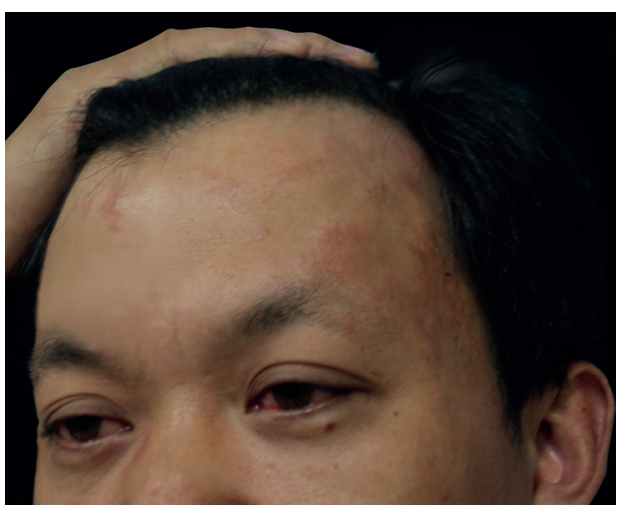

FiguRE 2: Bilateral eye conjunctival congestion

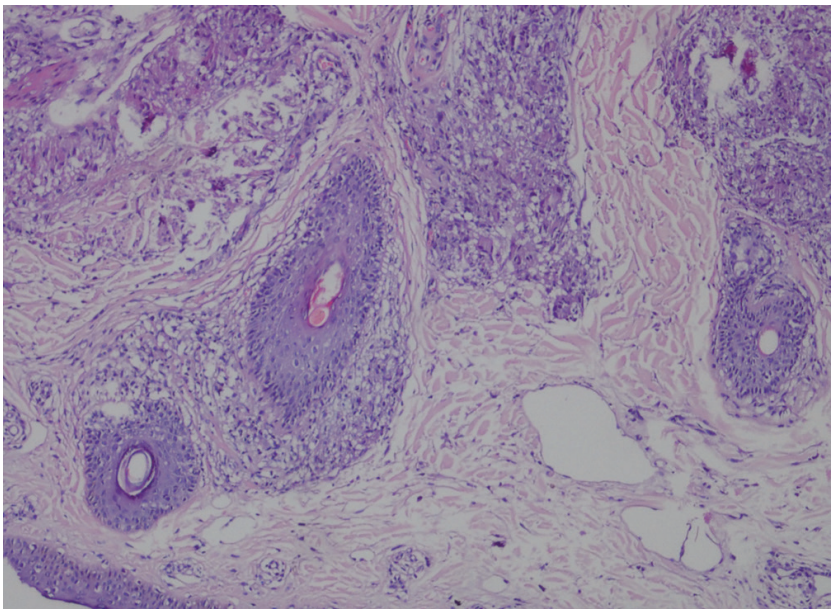

FIGURE 3: Histopathology of the skin lesion revealed a dermal inflammatory infiltrate composed mainly of epithelioid cells and a few multinucleated giant cells (Hematoxylin \& eosin x20)

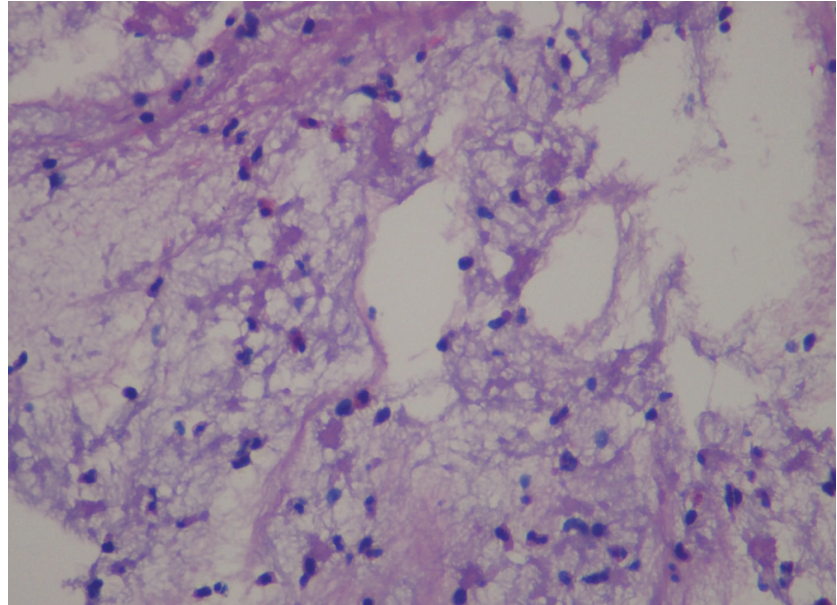

FIGURE 4: Mediastinal lymphadenopathy biopsy revealed the presence of lymphocytes and epithelioid macrophages (Hematoxylin \& eosin $\times 40)$

peared. The bilateral IOP measured normal. At a month follow-up, all skin lesions resolved. CT reexaminations revealed that bilateral hilar and mediastinal lymphadenopathy disappeared. Bilateral IOP remained normal. We prescribed the tapering of the initial dose of methylprednisolone $(24 \mathrm{mg} /$ daily) to $2 \mathrm{mg}$ / weekly on alternate days, which is the lowest dose that prevents relapse.

\section{DISCUSSION}

The patient described here presented to our department due to his skin lesions and ocular symptoms. Gaskin et al. ${ }^{6}$ reported the first case of neovascular glaucoma secondary to sarcoid-associated panuveitis in the absence of retinal ischaemia. Hamanaka et $a l .{ }^{7}$ demonstrated seven cases with secondary open-angle glaucoma due to suspected ocular sarcoidosis or confirmed sarcoidosis at the time of surgery. To our knowledge, open-angle glaucoma secondary to cutaneous sarcoidosis was uncommon in previous studies.

Cutaneous sarcoidosis is classified as either "specific" or "nonspecific". Specific skin lesions present as papules, plaques, subcutaneous nodules, lupus pernio, scar infiltration, alopecia, ulcerative lesions, and hypopigmentation among others. In general, specific skin lesions are usually associated with chronic sarcoidosis and reveal noncaseating granulomas in the dermis, subcutaneous fat, or both. Moreover, they show no correlation with the extent of systemic involvement, and do not indicate a more serious form of sarcoidosis. ${ }^{8}$ Nonspecific lesions include erythema nodosum, calcinosis cutis, prurigo, erythema multiforme, nummular eczema, nail clubbing, and Sweet syndrome. Nonspecific lesions are frequently associated with acute sarcoidosis and can not demonstrate sarcoidal granulomas. ${ }^{3,8}$

The classic histopathological picture of sarcoidosis is that of a noncaseating granuloma consisting of centrally organized collections of macrophages and epithelioid cells encircled by lymphocytes. ${ }^{2}$ However, a series of disease processes share the histopathological picture of a sarcoidal granuloma. Sarcoidosis is referred to as "the great imitator" clinically and histopathologically. When encountered with a biopsy showing a sarcoidal granuloma, clinicians 
should take into consideration diseases such as immunologic/foreign body granuloma (silica, tattoo granulomas), infectious diseases (tuberculosis, leprosy), neoplasias (breast and bronchial carcinomas, lymphoma), immunodeficiency disorders, drug eruptions, and other granulomatous processes (cheilitis granulomatosa, granulomatous rosacea, and Crohn's disease) ${ }^{8,9}$

There is no diagnostic test for sarcoidosis. Diagnosis relies on the integration of epidemiology, clinical manifestations, laboratory and radiographic examinations, and histopathology. ${ }^{8}$ The emphasis during physical examination and auxiliary examination should be placed on the skin, lungs, eyes, heart, and other organ systems. ${ }^{5}$ In particular, a tuberculin skin test is often performed in patients to rule out tuberculosis infection. Angiotensin-converting enzyme (ACE) levels may be used as an adjunct, but not for specific diagnosis. Radiographic diagnosis of sarcoidosis is done by chest radiography and cystic images. ${ }^{5}$

Our patient first visited our department due to his cutaneous manifestation and ocular symptoms. We recommended careful physical examination and auxiliary examination based on his clin-

\section{REFERENCES}

1. Collin B, Rajaratnam R, Lim R, Lewis H. A retrospective analysis of 34 patients with cutaneous sarcoidosis assessed in a dermatology department. Clin Exp Dermatol. 2010;35:131-4.

2. Haimovic A, Sanchez M, Judson MA, Prystowsky S. Sarcoidosis: a comprehensive review and update for the dermatologist: part II. Extracutaneous disease. J Am Acad Dermatol. 2012;66:719.e1-10.

3. Beutler BD, Cohen PR. Sarcoidosis in Melanoma Patients: Case Report and Literature Review. Cancers (Basel). 2015;7:1005-21.

4. Mana J, Marcoval J. Skin manifestations of sarcoidosis. Presse Med. 2012;41:355-74

5. Joseph C, Purvisha J, Patel BA. Sarcoidosis. J Am Acad Dermatol. 2005; 44: 725-45.

6. Gaskin BJ, Danesh-Meyer HV. Neovascular glaucoma and sarcoidosis. Eye (Lond). 2005;19:599-601.

7. Hamanaka T, Takei A, Takemura T, Oritsu M. Pathological study of cases with secondary open-angle glaucoma due to sarcoidosis. Am J Ophthalmol. 2002;134:17-26.

8. Fernandez-Faith $E$, McDonnell J. Cutaneous sarcoidosis: differential diagnosis. Clin Dermatol. 2007;25:276-87.

9. Ball NJ, Kho GT, Martinka M. The histologic spectrum of cutaneous sarcoidosis: a study of twenty-eight cases. J Cutan Pathol. 2004;31:160-8.

10. Ghabrial R, McCluskey PJ, Wakefield D. Spectrum of sarcoidosis involving the eye and brain. Aust N Z J Ophthalmol. 1997 ;25:221-4. ical manifestations. Significant examination results were not only bilateral hilar and mediastinal lymphadenopathy, skin and mediastinal lymphadenopathy biopsy findings, but also his elevated IOP. Studies suggested that the mechanism by which sarcoidosis usually results in elevated IOP may be associated with occlusion of the Schlemm's canal or fibrotic replacement of the canal. ${ }^{6,7}$

Ocular sarcoidosis may potentially lead to blindness. Consequently, all patients need eye examinations, even if they have no symptoms. ${ }^{5}$ Ocular sarcoidosis classically presents itself as acute anterior uveitis. Other ocular lesions include secondary glaucoma, posterior uveitis, conjunctival nodules, scleral plaques, lacrimal gland, and iritis. ${ }^{10}$

Treatment is essential for patients with progressive pulmonary disease, ocular disease, symptomatic cardiac dysfunction, and any organ involvement that adversely affects quality of life. Systemic corticosteroids are the most effective treatment for sarcoidosis. Topical corticosteroids are recommended for localized and mild disease limited to the skin. ${ }^{2}$ Other treatments include chloroquine, hydroxychloroquine, methotrexate, allopurinol, thalidomide, melatonin, tetracycline, and anti-TNF-alpha agents.., ${ }^{2,5}$

\author{
MAILING ADDRESS: \\ Jin-Hua Huang \\ Department of Dermatology \\ Third Xiangya Hospital \\ Central South University \\ Tongzipo Road, No. 139 \\ 410013 - Changsha, Hunan - China \\ E-mail:huangjinhua60@163.com
}

How to cite this article: Deng Q, Ding S, Yang SB, Huang JH. Cutaneous sarcoidosis and secondary open-angle glaucoma in a patient: case report and literature review. An Bras Dermatol. 2017;92(3):407-9. 